\title{
Evaluation of Fluorescent Probe Surface Intensities as an Indicator of Transdermal Permeant Distributions Using Wide-Area Two-Photon Fluorescence Microscopy
}

\author{
BETTY YU, ${ }^{1}$ KI HEAN KIM, ${ }^{2}$ PETER T.C. SO, ${ }^{2}$ DANIEL BLANKSCHTEIN, ${ }^{1}$ ROBERT LANGER ${ }^{1}$ \\ ${ }^{1}$ Department of Chemical Engineering, Massachusetts Institute of Technology, 77 Massachusetts Avenue, \\ Cambridge, Massachusetts 02139 \\ ${ }^{2}$ Department of Mechanical Engineering, Massachusetts Institute of Technology, Cambridge, Massachusetts 02139
}

Received 16 April 2002; revised 23 March 2003; accepted 29 April 2003

\begin{abstract}
The application of high-speed two-photon fluorescence microscopy (HTPM) to examine transdermal transport processes has enabled the noninvasive visualization of permeant spatial distributions over a larger, more clinically relevant wide area of the skin. Earlier studies demonstrated that the transdermal fluorescent probe distribution over a $2 \times 2 \mathrm{~mm}$ skin area was well represented by a significantly reduced sampling of the 400 microscale skin sites $(100 \times 100 \mu \mathrm{m})$ constituting the wide area. In the present study, the 400 microscale skin sites are considered individually, and the site-to-site variability in permeant distributions is used as a model to reflect the range in experimentally measured skin permeabilities resulting from the inherent stratum corneum structural heterogeneity. The correlation established between the permeant surface intensity and the corresponding permeant intensity gradient at each skin site provides an indication of the potential for screening transdermal permeant distributions solely based on the evaluation of microscale permeant surface intensities. The strong linear correlation between the intensity gradient and the surface intensity for the hydrophilic model permeant, sulforhodamine B, demonstrated that surface intensities provide a robust indicator of the corresponding transdermal probe distributions at the microscale. For the hydrophobic model permeant, rhodamine B hexyl ester, however, weak correlations were observed between these two parameters. This result suggests that the stratum corneum microscale surface intensity does not validly capture the corresponding intensity gradients for the entire range of skin permeabilities typically encountered as a result of the inherent stratum corneum heterogeneity. (c) 2003 Wiley-Liss, Inc. and the American Pharmacists Association J Pharm Sci 92:2354-2365, 2003
\end{abstract}

Keywords: transdermal; skin; fluorescence microscopy; percutaneous; permeability

\section{INTRODUCTION}

The transdermal route of drug delivery provides a noninvasive method of administering pharmaceutically relevant compounds over a sustained period. ${ }^{1,2}$ The inherent advantages exhibited by transdermal drug delivery for selected com-

Correspondence to: Daniel Blankschtein (Telephone: $617-$ 253-4594; Fax: 617-252-1651; E-mail: dblank@mit.edu)

Journal of Pharmaceutical Sciences, Vol. 92, 2354-2365 (2003)

(C) 2003 Wiley-Liss, Inc. and the American Pharmacists Association pounds over the more traditional methods of drug administration, including oral and subcutaneous, has stimulated the investigation of chemical enhancer systems that will facilitate the delivery of other drug candidates at therapeutically desired dosages. ${ }^{2-4}$ Recently, efforts to elucidate the mechanisms of chemical enhancer action have introduced the application of two-photon microscopy as an additional analysis technique. ${ }^{5-7}$ The 100-fold increase in skin-site imaging speed for transdermal applications using high-speed two-photon fluorescence microscopy (HTPM) has 
increased the efficiency with which the effects of various chemically induced changes in skin barrier properties can be examined for large sample sizes. ${ }^{8}$ Furthermore, recent findings have demonstrated that the wide-area average surface intensities and intensity gradients can be captured by a significantly reduced, representative number of skin sites. ${ }^{8}$ In this paper, the relationship between the model permeant surface intensity and the model permeant intensity gradient across the stratum corneum (SC) will be evaluated. Correlations between these two transport parameters enable the potential screening of transdermal permeant distributions induced by chemical enhancer action solely through the quantification of SC probe surface intensities. With respect to HTPM, these two parameters are captured by the model fluorescent permeant skin surface intensity and the corresponding intensity gradient. ${ }^{9}$ The oleic acid-induced changes in the spatial distribution of rhodamine $B$ hexyl ester (RBHE) and sulforhodamine B (SRB), the fluorescent model hydrophobic and hydrophilic drugs, respectively, were visualized and quantified throughout the thickness of the SC over a $4-\mathrm{mm}^{2}$ area of human cadaver skin (or a sample size of 400 different skin sites) using HTPM. ${ }^{9,10}$ Using the methodology described recently, ${ }^{6}$ the these two transport parameters were evaluated over 400 consecutive skin sites, where each skin site captures a $100 \times 100 \mu \mathrm{m}$ microscope field of view.

The rank-ordering of permeant surface intensities induced by different chemical enhancers to infer the corresponding transdermal permeant distributions will expedite the utilization of HTPM imaging to evaluate transdermal transport processes. In this way, the computationally intensive analyses previously performed to quantify the fluorescent probe spatial distributions over the wide area scanned can be circumvented. Moreover, the chemical enhancer-induced change in the microscale transport parameter correlation observed between these two transport parameters will provide additional insight into the mechanism of chemical enhancer action for each specific model permeant evaluated.

\section{Proposed Permeant Pathways}

The SC, the uppermost $10-20 \mu \mathrm{m}$ of the skin, serves as the primary barrier to transdermal transport of permeants. ${ }^{11}$ The SC has been described utilizing the brick and mortar model,,${ }^{11}$ in which the corneocytes (bricks) lie in a continuous phase of lipid multilamellae (mortar). Permeant partitioning into the SC, followed by permeant diffusion through the SC, are the two main steps in a simplified description of the permeation mechanisms across the SC. ${ }^{12}$ Because the lipid multilamellae of the intercorneocyte region form the only continuous phase through the SC, this intercellular region has been proposed to be the primary transdermal diffusion pathway for both hydrophilic and hydrophobic permeants. ${ }^{13-15}$ Within the intercellular transport region, however, the terms 'lipoidal' and 'aqueous pore' pathways have been used to describe the two different transport pathways that distinguish the mechanisms underlying the hydrophobic and the hydrophilic permeant transdermal transport, respectively. ${ }^{12,16}$

Lateral diffusion through the intercellular region was shown to constitute the rate-limiting step in transdermal transport for a series of hydrophobic probes in a model skin system of lipid multilamellar liposomes. ${ }^{14}$ However, the current understanding of the 'aqueous pore' pathway remains less developed, ${ }^{17}$ although the lacunar regions of the intercellular space have provided a morphological basis for the hydrophilic permeant transdermal transport pathway. ${ }^{18}$

For a uniform, homogeneous membrane, the flux, $J$, is described by the following equation:

$$
J=-D \frac{\mathrm{d} C}{\mathrm{~d} z}
$$

where $D$ is the membrane diffusion coefficient, and $\mathrm{d} C / \mathrm{d} z$ is the concentration gradient across the thickness of the membrane. The permeant flux is related to the membrane permeability by a constant, $\Delta C$, such that $P=J \Delta C$, where $\Delta C$ is the permeant concentration difference between the two media separated by the membrane. Because the permeability is also expressed as $P=K D / \mathrm{L}$, the flux is also directly related to $K$, the permeant vehicle-to-membrane partition coefficient. The membrane thickness is denoted by 1 . For the membrane described by eq. 1, the permeant membrane concentration gradient and the permeant vehicle to membrane partition coefficient are linearly related. These two transport parameters are captured in the HTPM intensity gradients and the surface intensities of the fluorescent model drugs, respectively. Moreover, the correlation between these two measures of probe spatial distribution in the SC provides an 
indicator of transport mechanisms, where deviations from a linear relationship suggest the presence of additional transport hindrance attributed to nonideal membrane transport. In the case of the hydrophobic permeant, deviations from linearity may be possibly attributed to the tortuous lipoidal pathway proposed earlier. ${ }^{19}$

\section{Skin Heterogeneity}

Although the SC membrane has been modeled as a uniform membrane in permeant transport studies, the inherent skin heterogeneity is illustrated in the HTPM wide-area scans by the siteto-site fluctuations of these two transport parameters over the 400 microscale skin sites imaged. For partitioning, the weakening of the SC barrier to enable increased permeant penetration can also be described by a decrease in $G^{*}$, the free energy associated with the work required for entry of a permeant into the SC lipid multilamellae. ${ }^{20,21} G^{*}$ can be related to the probe vehicle-to-skin partition coefficient, $K$, utilizing the following relation:

$$
K=\exp \left(\frac{-G^{*}}{R T}\right)
$$

where $R$ and $T$ are the gas constant $(8.314 \mathrm{~J} / \mathrm{mol}$ $\mathrm{K})$ and the absolute temperature of the system, respectively. ${ }^{20}$ According to eq. 2 , probe partitioning into the skin increases exponentially with decreases in the free energy, $G^{*}$. The difference between the free energy specific to one of the 400 skin sites examined and the 400 -skin site average free energy, $G_{\text {fluct }, i}$, can be determined using the following equation:

$$
\frac{G_{\text {fluct }, i}}{R T}=-\ln \left(\frac{K_{i}}{K_{\mathrm{A}}}\right)
$$

where $K_{i}$ is the partition coefficient at the individual skin site, $i$, and $K_{\mathrm{A}}$ is the global average vehicle-to-probe partition coefficient. In the studies reported here, $K_{i}$ and $K_{\mathrm{A}}$ are captured by the surface intensity at skin site $i$ and by the average surface intensity over the $4-\mathrm{mm}^{2}$ skin area surveyed, respectively. The subscript A can correspond to the control skin sample (C) or to the enhancer skin sample (E) for the cases of $K_{\mathrm{C}}$ and $K_{\mathrm{E}}$, respectively.

The individual skin site fluctuations in the probe intensity gradient $(\mathrm{d} I / \mathrm{d} z)_{\text {fluct }, i}$, reflect the fluctuations in the probe flux relative to the 400 -skin site average. Accordingly $(\mathrm{d} I / \mathrm{d} z)_{\text {fluct }, i}=(\mathrm{d} I / \mathrm{d} z)_{i}-$ $(\mathrm{d} I / \mathrm{d} z)_{\mathrm{A}}$, where $(\mathrm{d} I / \mathrm{d} z)_{i}$ is the intensity gradient at skin site $i$, and $(\mathrm{d} I / \mathrm{d} z)_{\mathrm{A}}$, is the 400 -skin site intensity gradient for which the subscript A refers to the control case (denoted by $\mathrm{C}$ ) or the enhancer case (denoted by E).

These fluctuations in the two transport parameters reflect the inherent skin heterogeneity, where variations in the SC structure over a skin sample induce a spread in surface intensity and intensity gradient distributions. This spread in the transport property values from skin site to skin site results from a spread in the SC structure and composition over the entire wide area scanned. The variations in the SC membrane flux at the macroscale have been well described in the literature, where intrasample variabilities in permeabilities up to $40 \%$ have been reported. ${ }^{22}$ The average wide-area transport properties arise from the individual transport properties measured for each of the 400 skin sites comprising the $4-\mathrm{mm}^{2}$ area scanned per skin sample.

By decomposing the wide area scanned into the $20 \times 20$ array of consecutive skin sites, the SC membrane can also be evaluated as a combination of microscale membranes whose inherent variability contribute to the visualized variations in the transdermal transport properties. The microscale distribution in transdermal permeant intensities captures a statistically significant spread of membrane permeabilities to model the spread in permeabilities that may arise from conducting 400 separate experiments, in which side-by-side diffusion cells are used. The specific microscale correlation obtained for each case examined is based on the 400 data points obtained from the 400 skin sites evaluated. The spread in permeabilities obtained by HTPM scanning provides a large database from which the validity of screening probe surface intensities to rank transdermal permeant distributions, and hence permeant flux, can be assessed.

\section{MATERIALS AND METHODS}

\section{Sample Preparation}

Full-thickness human cadaver skin from the abdominal area (National Disease Research Interchange, Philadelphia, PA), was kept frozen at $-80^{\circ} \mathrm{C}$ until use. Upon use, the skin was thawed at room temperature $\left(25^{\circ} \mathrm{C}\right)$, and the fat from the dermal side was removed. Skin samples of $2.25 \mathrm{~cm}^{2}$ were mounted in side-by-side diffusion cells (9 mm, PermeGear, Riegelsville, PA), and 
then immersed in phosphate buffered saline, PBS (Sigma, St. Louis, MO). Full-thickness skin was used instead of heat-stripped skin to preserve the structural features of the SC and the epidermis for the subsequent two-photon microscopy (TPM) image analysis.

Skin conductivities were measured in PBS before and after the diffusion cell experiments to ensure skin integrity before and after skin contact with the vehicle and the enhancer solutions. Ag$\mathrm{AgCl}$ electrodes (E242, In Vivo Metric, Healdsburg, CA) were connected to an AC signal generator (HP 33120A $15 \mathrm{MHz}$ Function Arbitrary Waveform Generator, Hewlett Packard, Palo Alto, CA) in series with a digital multimeter (Radio Shack ${ }^{\circledR}$, Fort Worth, TX). One electrode was placed in each compartment of the diffusion cell, which was filled with PBS, and a voltage drop of $100 \mathrm{mV}$ was applied across the skin. The current measurement was read from the multimeter and converted to an equivalent conductivity. The conductivity criterion of $>10 \mathrm{k} \Omega-\mathrm{cm}^{2}$ was applied, ${ }^{23}$ with skin samples not meeting this criterion replaced by intact skin.

The two fluorescent probes (Molecular Probes, Eugene, OR), rhodamine B hexyl ester perchlorate (RBHE) and sulforhodamine B (SRB), served as the hydrophobic and the hydrophilic model drugs, respectively, and were selected based on their similarity in molecular structure, disparity in octanol-water partition coefficient, and fluorescence emission range. The chemical structures of the two fluorescent probes are shown in Figure 1a,b, respectively, and their molecular weights (MW), fluorescence absorption (ab) and emission (em) spectral peaks (ab/em), and log $K_{\mathrm{O} / \mathrm{PBS}}$ (where $K_{\mathrm{O} / \mathrm{PBS}}$ is the experimentally determined octanol-PBS partition coefficient) values are listed in Table 1 . The $\log K_{\mathrm{O} / \mathrm{PBS}}$ values measured for RBHE and SRB are 2.49 and -0.45 , respectively. ${ }^{7}$ Under the $780 \mathrm{~nm}$ laser excitation, the fluorescence signals of the probes, their spectral $\mathrm{ab} / \mathrm{em}$ peaks at 556/578 $\mathrm{nm}$ (RBHE) and 565/586 $\mathrm{nm}$ (SRB), have minimal overlap with that corresponding to the skin autofluorescence, characterized by absorption and emission peaks of 488 and $515 \mathrm{~nm}$, respectively. ${ }^{24} \mathrm{SRB}$ and RBHE (Molecular Probes, Eugene, OR) probes were prepared at $0.33 \mathrm{mg} / \mathrm{mL}$ for both the control and the model chemical enhancer vehicles. The control vehicle consisted of $1: 1(\mathrm{v} / \mathrm{v})$ PBS and ethanol (Sigma, St. Louis, MO), with the chemical enhancer vehicle containing an additional 5\% (by volume) oleic acid (Sigma, St. Louis, MO). At a $\mathrm{pH}$ of 7.4, RBHE and SRB have net charges of +1 and zero, respectively.

The skin samples were removed from the diffusion cells following the 24-h skin exposure to the control and the enhancer fluorescent probe solutions. These skin samples were rinsed with PBS/ ethanol (1:1,by volume) to ensure removal of any excess fluorescent probes present on the surface of the skin, and then sealed in an imaging chamber (Coverwell $^{\mathrm{TM}}$, Grace Bio-Labs, Bend, OR) with a No. 1, 22-mm square coverslip (VWR Scientific, Media, PA) contacting the SC side. A drop of PBS was added to the chamber to prevent drying of the sample.

Contact between the SC and the coverslip was maximized to increase the consecutive number of TPM-imageable skin sites. Contact between the skin surface and the microscope slide coverslip was facilitated by first contacting the SC with the coverslip. Contact between the coverslip and the silicone spacer of the imaging chambers was then achieved to seal the skin sample in the imaging chamber. The thickness of the imaging chamber silicone spacer was selected to eliminate free space between the skin surface and the coverslip. Although these efforts to increase the uniformity of the inherently rough skin surface enabled the wide-area imaging of a majority of the consecutive

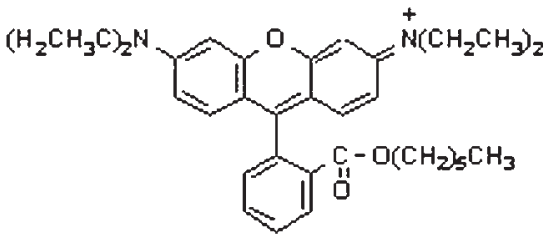

(a)

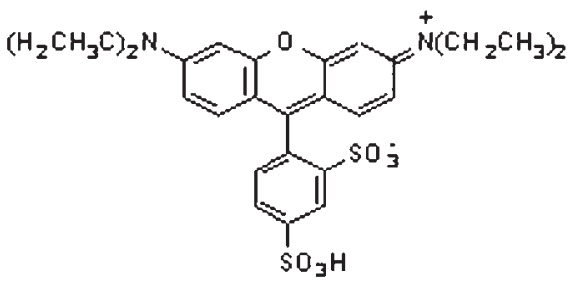

(b)

Figure 1. Hydrophobic and hydrophilic model drugs. The chemical structures are shown for (a) RBHE and (b) SRB. 
Table 1. Fluorescent Probe Properties of the Hydrophobic (RBHE) and the Hydrophilic (SRB) Model Probes Examined $^{a}$

\begin{tabular}{lcc}
\hline Probe & RBHE & SRB \\
\hline MW (Da) & 627 & 559 \\
ab/em (nm) & $556 / 578$ & $565 / 586$ \\
log $K_{\text {O/PBS }}{ }^{\circ}$ & $2.49 \pm 0.18$ & $-0.45 \pm 0.0045$ \\
\hline
\end{tabular}

${ }^{a}$ The chemical structures of RBHE and SRB are illustrated in Figure 1a,b, respectively; MW, molecular weight of each probe; $a b / e m$., the probe spectral absorption (ab) and emission peaks (em); log $K_{\mathrm{O} / \mathrm{PBS}}$ measured values of the log of the probe PBS-octanol partition coefficients (described in the Materials and Methods Section).

${ }^{b}$ Values are reported as mean $\pm \mathrm{SD}$. Experiments were performed in triplicate.

skin sites, skin sites not completely contacting the coverslip could not be imaged. These skin sites, where fluorescence intensity signals cannot be detected, were excluded from the analyses performed in this paper.

\section{High-Speed, Two-Photon Microscope and Data Analysis}

From our previous wide-area transdermal transport studies, ${ }^{9}$ the 400 -skin site probe surface intensity, $I_{z=0}$, and the 400 -skin site probe intensity gradient, $\mathrm{d} I / \mathrm{d} z$, were quantified for each of the four cases examined (RBHE-control, RBHEenhancer, SRB-control, and SRB-enhancer). For each skin site, or $100 \times 100 \mu \mathrm{m}$ area imaged, the skin surface was defined as the $z$ position corresponding to the highest site-specific average fluorescence intensity value. The fluorescence intensity gradient at each skin site was then calculated from the linear regression of the intensity profile over the first $10 z$-positions within the skin surface, starting with the defined surface $z$-position. Each subsequent $z$-position corresponds to an increment of $0.7 \mu \mathrm{m}$ into the depth of the SC. In the quantification of the wide-area skin surface intensity, the normalization of the skin surface $z$-position with respect to the maximum fluorescence intensity for each imaged skin site within the 400 consecutive skin sites scanned accounts for the skin surface roughness and for the skin site-to-skin site variability in the SC thickness. The average and median of the slope and of the intercept values that result from the 400 individual linear regressions performed corresponding to each skin site imaged are listed in Table 2.

To further quantify these fluctuations, the skin sites for which $G_{\text {fluct }, i}$ exceeded $1 R T$ were counted as maxima, whereas the number of minima corresponded to skin sites exhibiting $G_{\text {fluct }, i}$ values below $-1 R T$. The selection of \pm 1 $R T$ as the yardstick for determining the number of extrema reflects the thermal energy. As a basis of comparison, the thermal energy, or $1 R T$ per mole, roughly indicates the strength of an interaction, where interactions with energies exceeding $1 R T$ dominate over the disorganizing effects of thermal motion. ${ }^{25}$

For the intensity gradient fluctuations, maxima and minima were defined by the skin sites that exhibited intensity gradient values exceeding the range of values encompassed by the corresponding 400 -skin site intensity gradient value plus and minus the reported standard deviation (SD), respectively (see Table 2). Recall that for a normal distribution, 1 standard deviation, by definition,

Table 2. Central Tendencies of Transport Parameters ${ }^{a}$

\begin{tabular}{lccccc}
\hline & \multicolumn{2}{c}{ Intensity Gradient } & & \multicolumn{2}{c}{ Surface Intensity } \\
\cline { 2 - 3 } \cline { 5 - 6 } Drug & Mean & Median & & Mean & Median \\
\hline RBHE (C) & $57 \pm 36$ & 55 & & $551 \pm 281$ & 511 \\
RBHE (E) & $266 \pm 198$ & 240 & & $2546 \pm 2176$ & 1991 \\
SRB (C) & $6.9 \pm 8.9$ & 3.4 & & $84 \pm 90$ & 50 \\
SRB (E) & $67 \pm 56$ & 54 & & $838 \pm 467$ & 766 \\
\hline
\end{tabular}

\footnotetext{
${ }^{a}$ The intensity gradient and the surface intensity are represented, respectively, by the first-order (slope) and zero-order (intercept) linear regression parameters of the first 10 points in the linear region of the intensity profile generated at each of the 400 skin sites. The mean and the median values were calculated based on these individual linear regression results of 400 different sites in each sample. The control and enhancer samples are denoted by $(\mathrm{C})$ and (E), respectively; the mean is reported as the mean \pm SD.

${ }^{b}$ The absolute value of the intensity gradient is reported in units of counts/ $\mu \mathrm{m}$.

${ }^{\mathrm{c}}$ The surface intensity is reported in units of counts/pixel.
} 
includes $68.27 \%$ of the 400 intensity gradients measured. The number of maxima and minima determined for each skin sample examined, based on the application of the \pm 1 SD criteria to each of the 400 skin sites imaged, is given in Table 3. Note that the entries in Table 3 do not reflect a Gaussian distribution.

\section{RESULTS AND DISCUSSION}

\section{Probe Concentration Distribution in the Stratum Corneum}

The 400-skin site probe average intensity profiles, which reflect the probe concentration as a function of skin depth across the SC, are shown in Figure 2a,b for RBHE and SRB, respectively. In both Figure 2a,b, the solid and dashed lines indicate the average intensity for the control and the enhancer cases, respectively. The triangles and the squares depict the median intensities, all as a function of skin depth, for the control and the enhancer cases, respectively. For both the hydrophobic probe RBHE (Figure 2a) and the hydrophilic probe SRB (Figure 2b), the primary drop in intensity over the first $10-20 \mu \mathrm{m}$ for both the control and the enhancer cases corresponds well with the $10-20 \mu \mathrm{m}$ thickness of the SC. ${ }^{6}$ Linear regression of the linear region of the permeant intensity profiles was performed using the methodology introduced previously. ${ }^{6}$ The linear regressions of the profiles shown in Figure 2a,b yielded correlation coefficient of determination $\left(r^{2}\right)$ values of $0.96,0.98,0.99$, and 1.00 for RBHEcontrol, RBHE-enhancer, SRB-control, and SRBenhancer, respectively. These $r^{2}$ values indicate strong linear correlations between the probe intensity and the corresponding skin depth at each skin site imaged. The slope and the intercept reveal the magnitude of the permeant intensity gradient and the permeant surface intensity, respectively. A linear regression of the wide-area surface intensities and the corresponding widearea intensity gradients for the four cases examined yielded a $r^{2}$ value of 0.99 . The zero- and the first-order regression coefficients are 7.32 counts/ $\mu \mathrm{m}$ and $0.16 \mu \mathrm{m}^{-1}$, respectively. The good linear correlation observed between the permeant partitioning and the corresponding permeant concentration gradient within the SC membrane agrees with the uniform membrane model used to describe permeant permeabilities. Although the correlation between the surface intensity and the corresponding intensity gradient holds at the macroscale, the effect of reducing the SC area sampled on the relationship between these two transport parameters will be examined next.

\section{Oleic Acid-Induced Fluctuations in the Free Energy of Probe Partitioning}

The inherent skin heterogeneity and the distribution of transport parameters detected over the 400 consecutive skin sites imaged are depicted in Figures 3 and 4, respectively. At the global scale, the mean surface intensity and the mean intensity gradient calculated for each skin sample quantify the effects of chemical enhancer action on the respective two transport parameters. The effects of the SC morphology on the spatial variations in probe transport are visualized in Figure $3 \mathrm{a}-\mathrm{d}$, where response surfaces illustrate the relative fluctuations in the skin surface free energy of probe partitioning at each skin site, $G_{\text {fluct }, i} / R T$, defined in eq. 3 . The $z$-axis of Figure $3 \mathrm{a}-\mathrm{d}$ represents $G_{\text {fluct }, i} / R T$ as a function of the $x-y$ spatial position of the skin site imaged.

Table 3. Quantification of the 400-Skin Site Free Energy and Intensity Gradient Extrema

\begin{tabular}{lcccccc}
\hline & \multicolumn{2}{c}{ Free-Energy Fluctuation ${ }^{a}$} & & & Intensity-Gradient Fluctuation \\
\cline { 2 - 3 } \cline { 5 - 6 } Drug & No. of Maxima & No. of Minima & & No. of Maxima & No. of Minima \\
\hline RBHE (C) & 31 & 2 & & 52 & 69 \\
RBHE (E) & 92 & 28 & & 67 & 59 \\
SRB (C) & 113 & 34 & & 50 & 0 \\
SRB (E) & 29 & 4 & & 61 & 38 \\
\hline
\end{tabular}

\footnotetext{
${ }^{a}$ Maxima correspond to skin sites where $G_{\text {fluct }, i}>1 R T$, and minima correspond to skin sites where $G_{\text {fluct }, i}<-1 R T$. To quantify the number of maxima and minima, the activation energy fluctuation criterion of $1 R T$, the thermal energy, above and below the mean was employed.

${ }^{b}$ Maxima and minima are defined by skin sites exhibiting $|\mathrm{d} I / \mathrm{d} z|$ values exceeding the corresponding 400 -skin site average slope values shown in Table 1 by $+/-1 \mathrm{SD}$, respectively.
} 

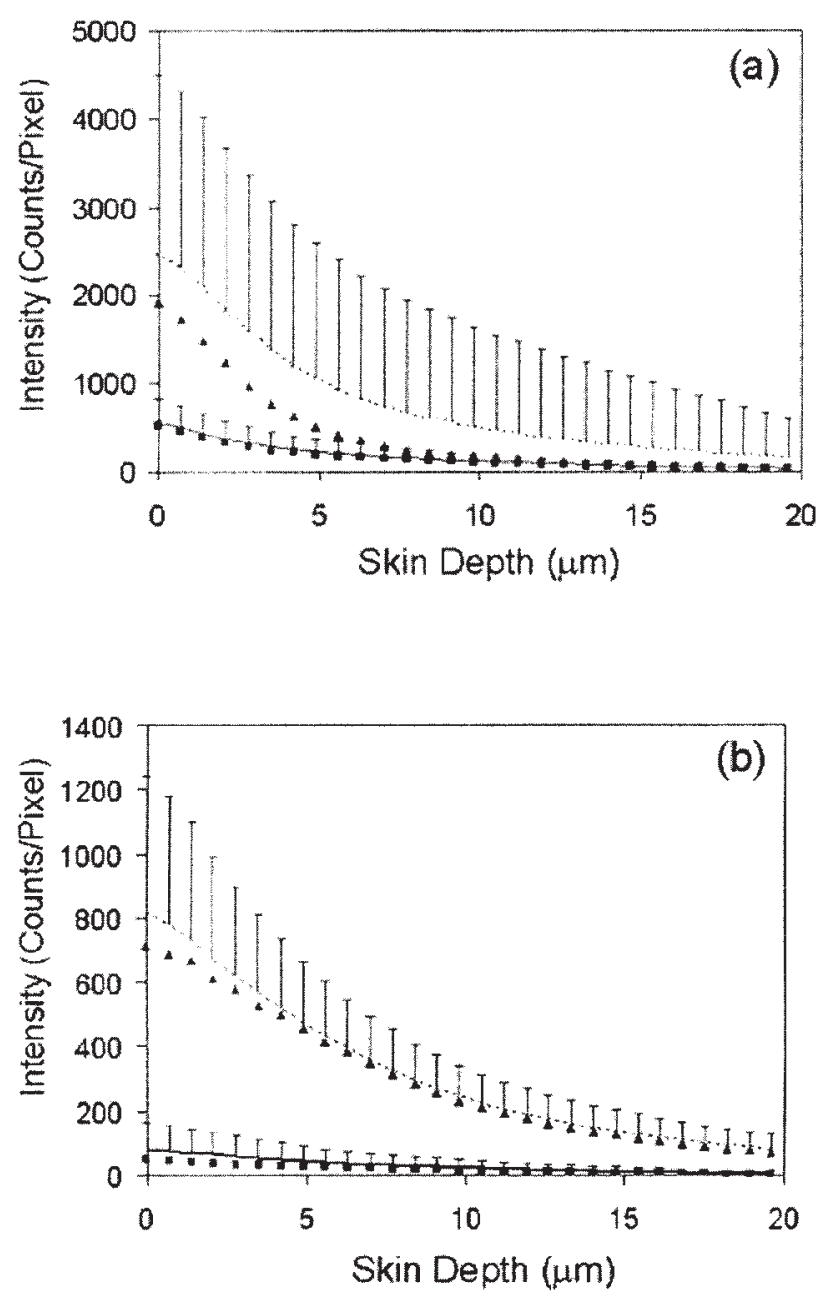

Figure 2. Probe intensity profiles. (a) RBHE. Key: wide-area average intensity (solid line, dotted line) and 400-skin site median intensity (squares, triangles) for the control and the enhancer samples, respectively. The average photon counts per pixel is plotted versus the corresponding skin depth. The error bars indicate $1 \mathrm{SD}$ from the wide-area average intensity of the 400 skin sites surveyed per sample. (b) SRB. Key: wide-area average intensity (solid line, dotted line) and wide-area median intensity (squares, triangles) for the control and the enhancer samples, respectively. The average photon counts per pixel is plotted versus the corresponding skin depth. The error bars indicate 1 SD from the wide-area average intensity of the 400 skin sites surveyed per sample.

Each unit on the $x y$ plane corresponds to $0.1 \mathrm{~mm}$. Surface intensities (measured at $z=0 \mu \mathrm{m}$ ) that yield $G_{\text {fluct }, i} / R T=0$ indicate that the corresponding skin site possesses a free energy of partitioning equal to the global average value. Hence, $G_{\text {fluct }, i} / R T=0$ reflects the globally detected skin barrier resistance to probe transport. From eq. 3 , the probe vehicle-to-skin partition coefficient, $K$, decays exponentially with increases in the probe free energy. Accordingly, in Figure $3 \mathrm{a}-\mathrm{d}$, the peaks correspond to skin sites with higher than average barrier resistance (smaller $K$ values) and the valleys reflect high permeant transport regions (larger $K$ values). The 3 -dimensional surfaces in Figure 3a-d map the fluctuations in the free energy over the SC topology that are responsible for the skin barrier function. The number of maxima, or peaks, increased from 31 to 92 , and the number of minima, or valleys, increased from 2 to 28 as a result of the oleic acid enhancer action (see Table 3).

For the hydrophilic probe SRB, however, the control case exhibits a large total number of extrema, with 113 maxima and 34 minima. The inherently low permeability of the SC to the hydrophilic probe that is revealed in the low probe partitioning can be further visualized over the area defined by $x=0-20$ units and $y=0-10$ units in Figure 3c. Here, the existence of higher SRB free energies (positive deviations from $G_{\text {fluct }} / R T=0$ ) over a significant region of the total $4 \mathrm{~mm}^{2}$ for the control case suggests that transport of the hydrophilic probe is limited to a small number of higher transport regions encompassed by the energy minima shown in Figure 3c. Out of the 400 skin sites examined, $28 \%$ of the total skin area manifests free energy barriers exceeding the 400 -skin site average by $1 R T$, the criterion established earlier. Compared with the 113 maxima, the 34 free energy minima that lie $1 R T$ below the sample average comprise $8.5 \%$ of the total $4-\mathrm{mm}^{2}$ skin area examined. These domains of higher transport remain consistent with the proposed existence of 'aqueous pore' pathways through which hydrophilic probes can traverse the lipoidal SC matrix as a result of inherent imperfections in the SC lipid multilamellae.

In the presence of oleic acid (see Figure 3d), the number of peaks decreases from 113 to 29 and the number of valleys decreases from 34 to 4 . These decreases in the number of extrema signal the increased uniformity in probe transport, possibly resulting from the enhancer-induced formation of additional hydrophilic probe pathways. The SC barrier response to the oleic acid enhancer action is displayed in Figure 3d, where the reduction in the skin sample free energy, evident in the 10-fold increase in the 400-skin site surface intensity, enables an increased probability of probe partitioning resulting from an increase in the number of 

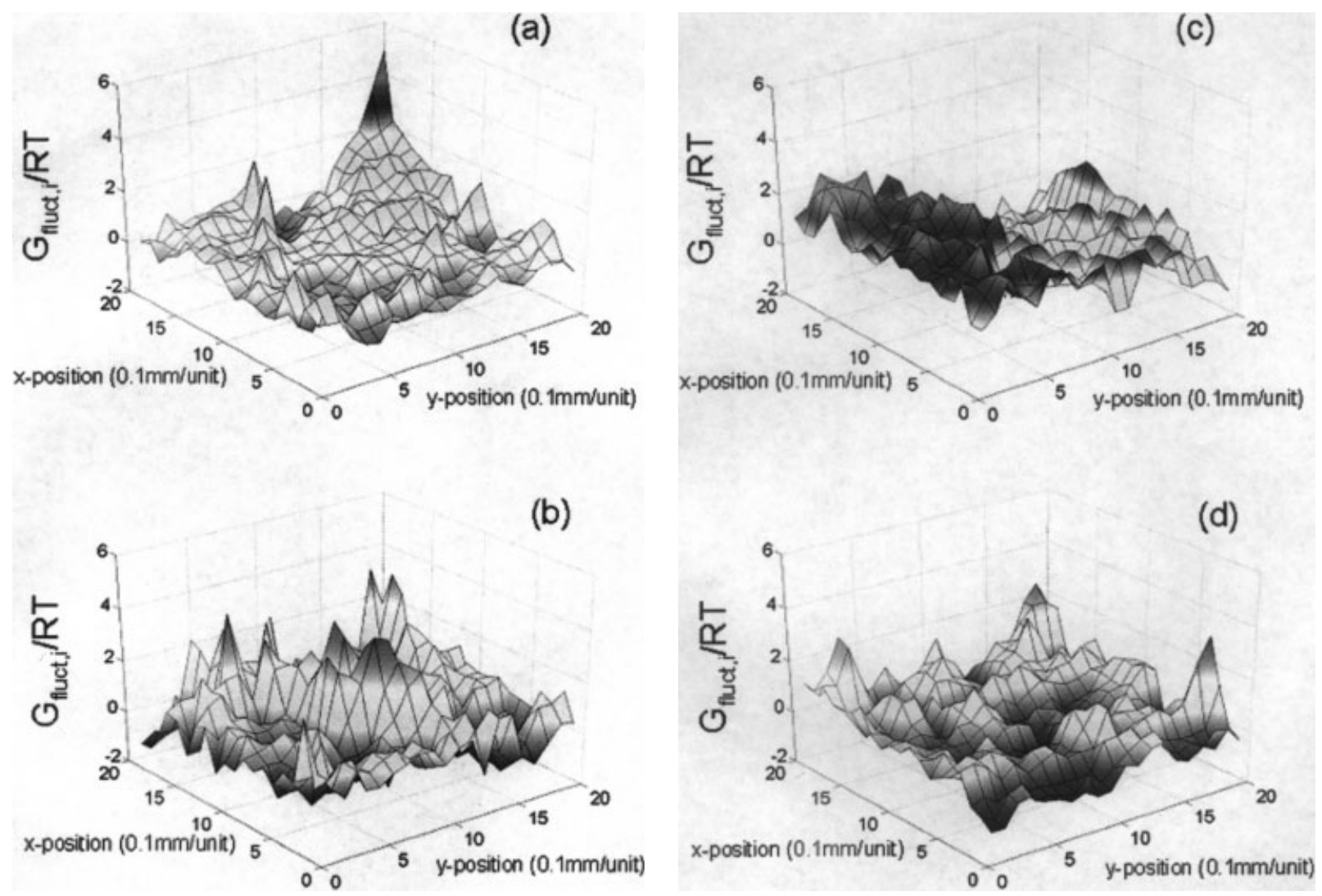

Figure 3. Spatial fluctuations in free energy. Surface response plots illustrate $G_{\text {fluct }, i} /$ $R T$ (represented on the $z$-axis), defined in eq. 3, as a function of the spatial coordinates displayed in the $x-y$ plane for (a) RBHE-control, (b) RBHE-enhancer, (c) SRB-control, and (d) SRB-enhancer. Each unit along the $x$ - and the $y$-axes corresponds to a length of $0.1 \mathrm{~mm}$, such that the total $4-\mathrm{mm}^{2}$ skin area is depicted.

skin sites possessing free energies that lie within $+/ 1 R T$ of the sample average.

\section{Oleic Acid-Induced Fluctuations in the Permeant Intensity Gradient}

Figure $4 a-d$ show the fractional fluctuations in the intensity gradient over the first $7 \mu \mathrm{m}$ of the SC corresponding to the spatial position of each skin site scanned. The $z$-axis depicts the normalization of $(\mathrm{d} I / \mathrm{d} z)_{\text {fluct }, i}$ by $(\mathrm{d} I / \mathrm{d} z)_{\mathrm{A}}$ corresponding to each skin site, whose spatial coordinates are depicted by the $x-y$ coordinates.

\section{Hydrophobic Probe}

The oleic acid-induced changes in the intensity gradient fluctuations reported in Table 3 indicate minor increases in the total number of extrema for the hydrophobic probe, with $121=52+69$ extrema for the control case and $126=67+59$ extrema for the enhancer case. The corresponding surface plots in Figure 4a,b further illustrate the similarity in the intensity gradient fluctuations between the control and the enhancer cases of the hydrophobic probe, respectively.

For the hydrophobic probe, oleic acid induced relatively minor increases for the intensity gradient fluctuations observed over the $4-\mathrm{mm}^{2}$ skin area examined, with the extrema shifting from 121 to 126 (see Table 3). The insensitivity of the intensity gradient fluctuations in response to the oleic acidinduced increases in the free energy fluctuations indicates a weak correlation between the surface intensity and the intensity gradients for the hydrophobic probe for significantly reduced membrane areas. This observation may be attributed to the earlier finding that lateral diffusion is the ratelimiting transport step for hydrophobic model permeants. ${ }^{14}$ 

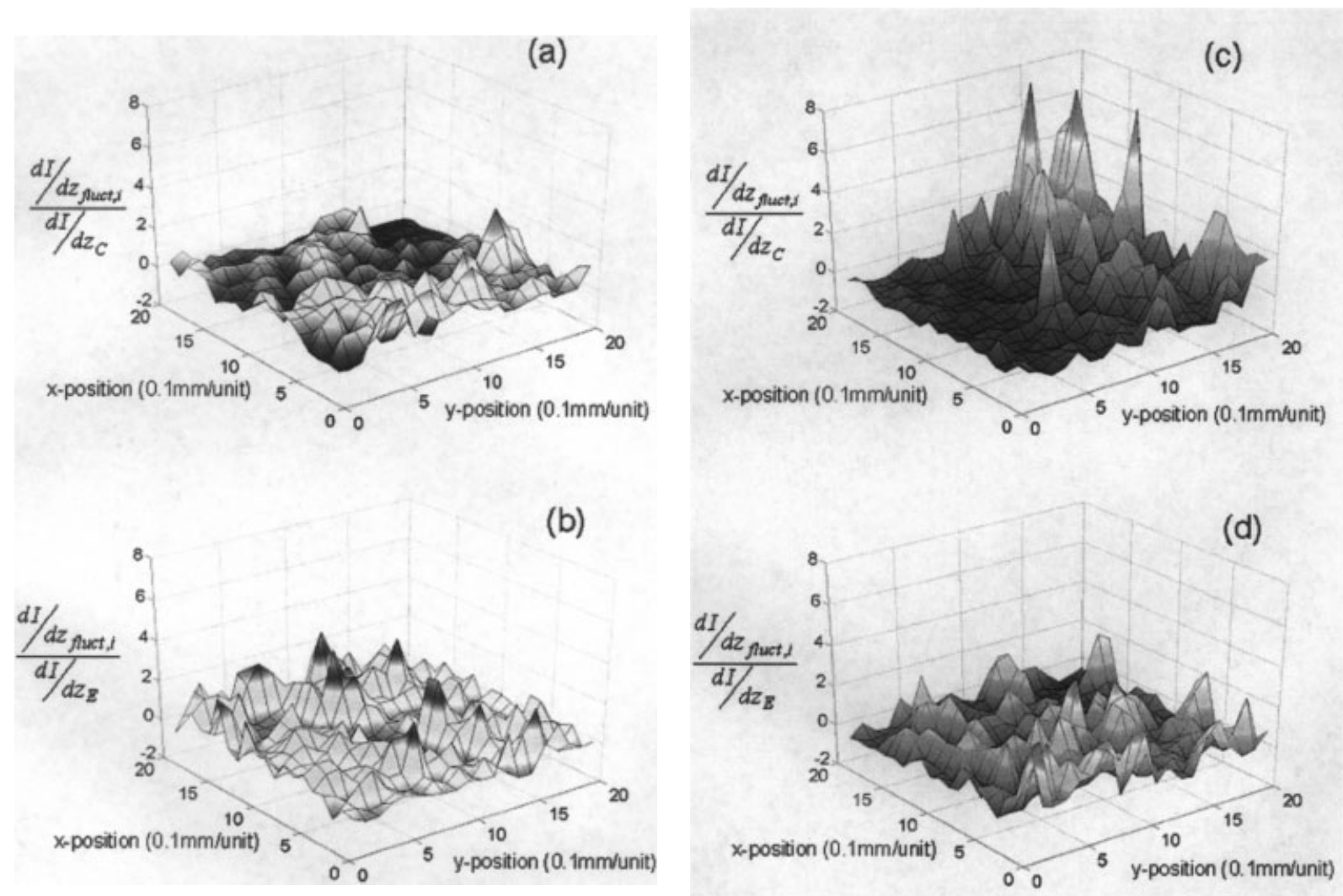

Figure 4. Spatial fluctuations in probe concentration gradient. Surface response plots illustrate $\mathrm{d} I / \mathrm{d} z_{\text {fluct }, i}$ (represented on the $z$-axis), as a function of the spatial coordinates displayed in the $x-y$ plane for (a) RBHE-control, (b) RBHE-enhancer, (c) SRB-control, and (d) SRB-enhancer. Each unit along the $x$ - and the $y$-axes corresponds to a length of $0.1 \mathrm{~mm}$, such that the total $4-\mathrm{mm}^{2}$ skin area is depicted.

\section{Hydrophilic Probe}

The intensity gradient fluctuations for the control case (Figure 4c) and for the enhancer case (Figure 4d) of the hydrophilic permeant indicate that oleic acid induced increases in the number of fluctuations. These significant increases in the total number of fluctuation extrema resulting from the chemical enhancer action are shown in Table 3. The number of extrema increases from 55 to 99 , from the control to the enhancer sample, whereas the number of free energy fluctuations extrema decreases from 147 to 33 (see Table 3). The strong correlation between the decrease in free energy fluctuations and the increase in the intensity gradient fluctuations suggests that oleic acid-induced changes in the hydrophilic probe vehicle-to-skin partitioning step of transport remains the rate-limiting step.

The correlation between the fluctuations in the microscale intensity gradient and the correspond- ing surface intensity at each of the 400 skin sites is quantified next.

\section{Correlation between Model Drug Vehicle-to-Skin Partitioning and Intensity Gradient}

The good correlation $\left(r^{2}=0.99\right)$ between the widearea permeant surface intensity and the corresponding wide-area intensity gradient suggests that, at the global scale, the surface intensity provides an indication of transmembrane permeant distributions. For the permeant molecular weights and the hydrophilicities examined here, the linear relationship holds in the presence of chemical enhancer action and appears to be independent of the permeant physicochemical properties. In light of the fluctuations in each transport parameter underlying the mean transport parameter values obtained (see Figures 3 and 4), the applicability of this linear relationship at the microscale will be examined next. Specifically, 
the correlation between the permeant intensity gradient and the permeant surface intensity at each of the 400 skin sites will be determined. In contrast to the wide-area linear relationships that characterize transport across the SC membrane, the regression coefficients determined from the site-specific HTPM data assess the validity of the uniform membrane model on a significant reduction in the sample size from 4 to $0.01 \mathrm{~mm}^{2}$.

The relationships between the model drug surface intensity and the corresponding intensity gradient within each wide-area scanned are depicted in Figure $5 \mathrm{a}-\mathrm{d}$. These two values reflect the model drug vehicle-to-skin partitioning and the model drug skin concentration gradient, respectively. For a model membrane, the model drug vehicle-to-skin partitioning is directly pro- portional to the drug flux, which in this paper, is evaluated with the model drug intensity gradient. The linear regression results obtained for each of the plots shown in Figure 5a-d, where the straight line indicates the best linear fit of the data shown by the triangles, are tabulated in Table 4 . The slope and the intercept are indicated by the first- and zero-order linear regression coefficients, respectively. Furthermore, their 5\% confidence intervals are indicated following each reported value. The strength of the linear correlation is revealed by the $r^{2}$ values shown in the last column of Table 4 .

For the hydrophobic probe, the decrease in the correlation between the skin site surface intensities and the intensity gradients as a result of oleic acid enhancer action is observed in the $r^{2}$ values of 0.77 and 0.57 , for the control and the enhancer
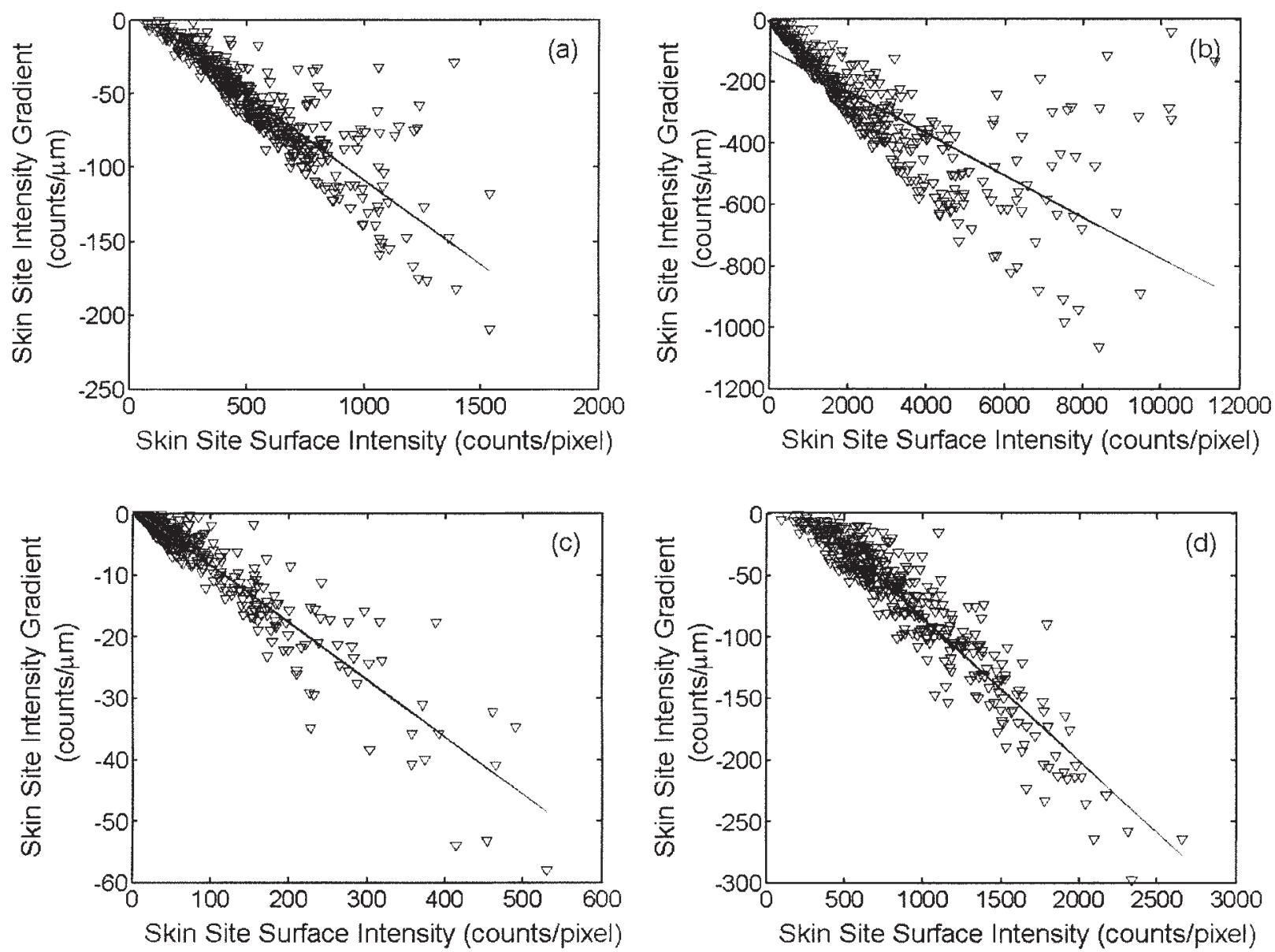

Figure 5. Model drug surface intensity and intensity gradient correlation. The diamonds correspond to the skin site intensity gradients as a function of the corresponding surface intensities for (a) RBHE-control, (b) RBHE-enhancer, (c) SRB-control, and (d) SRB-enhancer. The solid line in each panel depicts the best-fit line resulting from the linear regression analyses of each data set. See Table 4 for the zero- and first-order linear regression coefficients, as well as for the $r^{2}$ value corresponding to each data set. 
Table 4. Skin Site Surface Intensity-Intensity Gradient Correlation: Linear Regression Coefficients ${ }^{a}$

\begin{tabular}{lcrr}
\hline & $\begin{array}{c}\text { First-Order } \\
\text { Coefficient } \\
\left(\times 10^{-3}\right)\end{array}$ & $\begin{array}{r}\text { Zero-Order } \\
\text { Coefficient }\end{array}$ & $r^{2}$ \\
\hline Drug & $-114 \pm 6.19$ & $5.71 \pm 3.85$ & 0.77 \\
RBHE (C) & $-68.0 \pm 6.00$ & $-98.0 \pm 20.6$ & 0.57 \\
SRB (C) & $-93.2 \pm 3.38$ & $0.89 \pm 0.42$ & 0.88 \\
SRB (E) & $-116 \pm 4.21$ & $31.3 \pm 4.16$ & 0.89 \\
\hline
\end{tabular}

${ }^{a}$ Linear regressions were performed for the data shown in Figure $5 \mathrm{a}-\mathrm{d}$, where the surface intensity was plotted versus the corresponding intensity gradient for each skin site imaged per skin sample. The first-order and zero-order linear regression coefficients reflect the slope and the intercept of these plots. The corresponding $5 \%$ confidence interval is reported following the value of each linearly regressed coefficient. The $r$-square values that indicate the goodness of fit, or the strength of the correlation between the surface intensity and the intensity gradient, are shown in the final column.

cases, respectively. Comparing the data shown in Figure 5a with those seen in Figure 5b, increased deviations from the best-fit line are observed in the presence of oleic acid. For both the control and the enhancer cases, the deviations in the transport parameter data from linearity occur at skin sites possessing higher surface intensities. In the presence of the enhanced transport, the deviation from linearity becomes exaggerated, as indicated by the decreased $r^{2}$ value of 0.57 . The action of oleic acid on increasing hydrophobic permeant solubility in the lipid multilamellar pathway has recently been described using dual-channel HTPM. ${ }^{7}$ This observation may arise from the transport hindrance imposed on the permeant as a result of the tortuous lipoidal pathway characterizing lateral diffusion. These deviations from ideal membrane behavior that are observed through the HTPM microscale correlations provide insight into the mechanisms of transport that are not observed with wide area observations. Using the 400 -site SC micromembrane model of the wide-area scanned, the variability in the intensity gradients can be attributed to corresponding surface intensities with a confidence level of $77 \%$ for the hydrophobic control case. The validity of the uniform membrane model for the hydrophobic probe diminishes in the presence of chemical enhancer action, where the intensity gradients can be explained by the corresponding surface intensities only $57 \%$ of the time. These figures arise from the coefficient of determination, or $r^{2}$, values of 0.77 and 0.57 , respectively. The weak linear correlation between the surface intensity and the corresponding intensity gradient over the 400 skin sites examined suggests that surface intensities may not provide a robust indicator of the corresponding permeant concentration gradient over the entire spectrum of SC permeabilities.

The linear relationship between the permeant surface intensity and the permeant intensity gradient that is characteristic of ideal membrane transport is maintained at the microscale for the hydrophilic permeant. The good correlation between the hydrophilic model drug surface intensity and the corresponding intensity gradient for the control case is revealed by the $r^{2}$ value of 0.88 . In the presence of chemically induced skin barrier alterations, the strong correlation between partitioning and drug intensity gradient remains, as indicated by the $r^{2}$ value of 0.89 for the hydrophilic enhancer case. Hence, increases in the hydrophilic drug intensity gradient correspond well with the hydrophilic drug intensity increases observed at the skin surface. The linear correlation observed between permeant surface intensity and the corresponding intensity gradient over the 400 different skin sites evaluated reveals that the microscale hydrophilic surface intensities provide a confident indicator of transdermal permeant spatial distributions quantified by the intensity gradient. Furthermore, the specific coefficient of determination obtained for each case considered ( 0.88 and 0.89 for the control and the enhancer, respectively), indicates that the linear correlation remains valid for the hydrophilic permeant in the presence of SC variability.

\section{CONCLUSIONS}

The use of HTPM has enabled the visualization of transdermal transport parameters over a wide area of SC. The good linear fit obtained for the wide-area surface intensity and corresponding wide-area intensity gradient for both the hydrophobic and the hydrophilic model permeants agrees well with transport across a uniform membrane. The evaluation of the correlation between the surface intensity and the intensity gradient over the 400 individual sites that constitute the wide-area imaged captures the spread in permeant distributions that may reflect the sampleto-sample permeability heterogeneity that is well described in the literature. For the hydrophobic model permeant, the permeant surface intensity is not a reliable indicator of the corresponding 
intensity gradient. However, the strong correlation established between the hydrophilic probe surface intensity and the corresponding intensity gradient suggests that the hydrophilic probe surface intensities resulting from chemical enhancer action may be ranked to potentially screen the efficacy of a myriad chemical enhancer formulations. This application of HTPM as a screening tool warrants additional examination.

\section{ACKNOWLEDGMENTS}

The authors thank Professor Michael Holick, Professor Sidney Yip, and Professor Martin Bazant for helpful discussions regarding the analyses of transport data. The authors also thank the National Institutes of Health grant GM 44884 for support of this work.

\section{REFERENCES}

1. Guy RH. 1996. Current status and future prospects of transdermal drug delivery. Pharm Res 13:17651769.

2. Naik A, Kalia YN, Guy RH. 2000. Transdermal drug delivery: Overcoming the skin's barrier function. PSTT 3:318-326.

3. Finnin BC, Morgan TM. 1999. Transdermal penetration enhancers: Applications, limitations, and potential. J Pharm Sci 88:955-958.

4. Fuhrman LC, Michniak BB, Behl CR, Malick AW. 1997. Effect of novel penetration enhancers on the transdermal delivery of hydrocortisone: An in vitro species comparison. J Controlled Release 45:199206.

5. Yu B, Kim K, So PTC, Blankschtein D, Langer R. 2002. Topographic heterogeneity in transdermal transport revealed by high-speed two-photon microscopy: Determination of representative skin sample sizes. J Invest Dermatol 118: 1085-1088.

6. Kim KH, Buehler C, So PTC. 1999. High-speed, two-photon scanning microscope. Appl Optics 38: 6004-6009.

7. Yu B, Dong C-Y, So PTC, Blankschtein D, Langer R. 2001. In vitro visualization and quantification of oleic acid induced changes in transdermal transport using two-photon fluorescence microscopy. J Invest Dermatol 117:16-25.

8. Huzaira M, Rius F, Rajadhyaksha M, Anderson RR, González S. 2001. Topographic variations in normal skin, as viewed by in vivo reflectance confocal microscopy. J Invest Dermatol 116:846-852.
9. Williams AC, Cornwell PA, Barry BW. 1992. On the non-Gaussian distribution of human skin permeabilities. Int J Pharm 86:66-77.

10. Southwell D, Barry BW, Woodford R. 1984. Variations in permeability of human skin within and between specimens. Int J Pharm 57:299-309.

11. Kasting GB, Smith RL, Cooper ER. 1987. Effect of lipid solubility and molecular size on percutaneous absorption. Pharmacology of the skin, Basel: Karger, pp. 138-153.

12. Noonan PK, Gonzalez MA. 1990. Pharmacokinetics and the variability of percutaneous absorption. J Toxicol Cut Ocular Toxicol 8:511-516.

13. Liu PC, Nightingale JAS, Kuriharabergstrom T. 1993. Variation of human skin permeation in vitro Ionic vs neutral compounds. IntJ Pharm 90:171-176.

14. Schaefer H, Redelmeier TE. 1996. Skin barrier. 1. Basel: Karger, p. 310.

15. Sznitowska M, Berner B, Maibach HI. 1995. Percutaneous penetration of multipolar ions-Evidence for porous transport. Int J Pharm 123: 41-45.

16. Suhonen TM, Bouwstra JA, Urtti A. 1999. Chemical enhancement of percutaneous absorption in relation to stratum corneum structural alterations. J Controlled Release 59:149-161.

17. Johnson ME, Blankschtein D, Langer R. 1997. Evaluation of solute permeation through the stratum corneum: Lateral bilayer diffusion as the primary transport mechanism. J Pharm Sci 86:11621172.

18. Potts RO, Guy RH. 1992. Predicting skin permeability. Pharm Res 9:663-669.

19. Manabe E, Sugibayashi K, Morimoto Y. 1996. Analysis of skin penetration enhancing effect of drugs by ethanol-water mixed systems with hydrodynamic pore theory. Int J Pharm 129:211-221.

20. Sznitowska M, Janicki S, Williams AC. 1998. Intracellular or intercellular localization of the polar pathway of penetration across stratum corneum. J Pharm Sci 87:1109-1114.

21. Menon GK, Elias PM. 1997. Morphologic basis for a pore-pathway in mammalian stratum corneum. Skin Pharmacol 10:235-246.

22. Garrison MD, Doh LM, Potts RO, Abraham W. 1994. Effect of oleic acid on human epidermis: Fluorescence spectroscopic investigation. J Controlled Release 31:263-269.

23. Golden GM, McKie JE, Potts RO. 1987. Role of stratum corneum lipid fluidity in transdermal drug flux. J Pharm Sci 76:25-28.

24. Green PG, Guy RH, Hadgraft J. 1988. In vitro and in vivo enhancement of skin permeation with oleic and lauric acids. Int J Pharm 48:103-111.

25. Mak VHW, Potts RO, Guy RH. 1990. Oleic acid concentration and effect in human stratum corneum: Non-invasive determination by attenuated total reflectance infrared spectroscopy. J Controlled Release 12:67-75. 